\section{Should Hospital Admission Be Used as an Opportunity for Deprescribing in Older Adults?}

\section{THE "PRO" SIDE}

Polypharmacy, defined as the use of 5 or more medications, is becoming increasingly common in older adults, internationally. For example, in a Canadian survey of experiences with primary health care, $27 \%$ of older adults reported taking 5 or more medications on a regular basis. ${ }^{1}$ Polypharmacy is associated with medication-related adverse effects such as frailty, disability, death, and falls. ${ }^{2}$ Deprescribing-the process of withdrawing an inappropriate medication, under the supervision of a health care professional, with the goal of managing polypharmacy and improving outcomes-may be a solution to reduce the harm associated with using multiple medications. ${ }^{3}$ Evidence is accumulating to suggest that initiating deprescribing interventions within the hospital setting can be feasible, safe, and sustained after discharge. For patients with polypharmacy, admission to hospital can give clinicians an opportunity to reassess medications, identify the risks and harms of the current medication regimen, and initiate deprescribing of inappropriate medications, because the necessary resources, time, and specialist health care practitioners are often readily available in this setting. Hospitals also represent a somewhat "controlled" environment, where clinicians can closely monitor and reassess patients after implementing deprescribing interventions. To evaluate whether hospitalization should be used as an opportunity for deprescribing, the effectiveness of hospital-based deprescribing interventions must be analyzed.

A recent systematic review of randomized trials evaluating the impact of deprescribing interventions on older adults in hospital demonstrated that such interventions are safe, feasible, and generally effective in reducing potentially inappropriate medications. ${ }^{4}$ Since publication of this systematic review, many other studies have provided additional evidence to support the proposition that hospitalization offers an opportunity for deprescribing in older adults.

In a single-arm interventional study, hospitalized Canadian patients aged 65 years or older, who were longterm regular users of sedative medications, received a selfdirected patient education pamphlet describing the risks of prolonged use of sedatives and outlining a stepwise tapering protocol. ${ }^{5}$ These hospitalized older adults were willing to discontinue their sedative medications, and of the 50 participants enrolled in the study, 32 (64\%) had successful deprescribing of their sedative medication in hospital, with no reported episodes of acute withdrawal. Importantly, the study found no change in self-reported sleep disturbances after the hospital stay (relative to preadmission occurrences), which indicates that the intervention was feasible and safe.

In another study, conducted in Australia, McKean and others ${ }^{6}$ investigated whether a structured approach to deprescribing was feasible and whether it reduced medication burden. A sample of 50 hospital inpatients aged 65 years or older underwent a deprescribing intervention, which included an education program targeted toward clinicians and implementation of a 5-step decision support tool for selecting eligible medications for discontinuation. ${ }^{7}$ The intervention resulted in a significant decrease in the median number of medications per patient at discharge. At follow-up, less than $5 \%$ of ceased medications were recommenced, and this occurred among less than $10 \%$ of the patients. There were no deaths or acute presentations to hospital attributable to ceasing the medications. These findings demonstrate that a multifactorial hospital intervention can lead to safe and successful deprescribing of inappropriate medications in older adults. Similarly, a study conducted in an Australian tertiary hospital evaluated the feasibility of a pharmacist-led, physician-supported deprescribing model, in which patients 65 years or older with polypharmacy were evaluated for deprescribing by team pharmacists. ${ }^{8}$ In that study, $60 \%$ of patients had successful deprescribing of inappropriate medications, which showed that this model of deprescribing in an acute hospital setting is feasible and that deprescribing is becoming an essential role for clinical pharmacists. ${ }^{9}$

A further example involved a prospective dual-arm interventional study conducted in a Canadian tertiary care hospital. ${ }^{10}$ The study aimed to reduce the number of medications prescribed at hospital discharge following pharmacist-led, patient-specific deprescribing rounds for inpatients. The deprescribing rounds resulted in significantly more medications being deprescribed relative to the control, with a significant reduction in rates of hospital readmission and presentations to the emergency department.

There is also some evidence to suggest that not initiating deprescribing interventions in hospital may be a missed opportunity to improve medication use in older adults. In the United Kingdom, a study to quantify and describe the nature of deprescribing in a teaching hospital found limited deprescribing activity, dominated by reactive behaviour from clinicians (such as a response to an adverse clinical trigger), as opposed to proactive efforts to deprescribe inappropriate medications. ${ }^{11}$ Similarly, in a Canadian study, ${ }^{12}$ the rates of use and discontinuation of docusate sodium and 
other laxatives by internal medicine inpatients was documented; the investigators found that docusate was frequently and inappropriately prescribed to hospital inpatients, with approximately $80 \%$ of patients continuing docusate use at the time of discharge. These results demonstrate that deprescribing interventions are needed within hospital settings to reduce inappropriate use of medications.

Overall, the growing evidence from systematic reviews and interventional studies suggests that hospitalization may be a good opportunity to initiate deprescribing interventions for older adults. Often, deprescribing needs to be actively promoted to health care practitioners and patients, with the message that it should not be considered as an isolated task, but rather forms part of a comprehensive medication management review for older adults. ${ }^{13}$ The patient's or caregiver's goals and attitudes to their health and medications should always be considered before commencing any deprescribing interventions.

\section{References}

1. Reason B, Terner M, Moses McKeag A, Tipper B, Webster G. The impact of polypharmacy on the health of Canadian seniors. Fam Pract. 2012;29(4):427-32.

2. Gnjidic D, Hilmer SN, Blyth FM, Naganathan V, Waite L, Seibel MJ, et al. Polypharmacy cutoff and outcomes: five or more medicines were used to identify community-dwelling older men at risk of different adverse outcomes. J Clin Epidemiol. 2012;65(9):989-95.

3. Reeve E, Gnjidic D, Long J, Hilmer S. A systematic review of the emerging definition of 'deprescribing' with network analysis: implications for future research and clinical practice. Br J Clin Pharmacol. 2015;80(6):1254-68.

4. Thillainadesan J, Gnjidic D, Green S, Hilmer SN. Impact of deprescribing interventions in older hospitalised patients on prescribing and clinical outcomes: a systematic review of randomised trials. Drugs Aging. 2018;35(4):303-19.

5. Wilson MG, Lee TC, Hass A, Tannenbaum C, McDonald EG. EMPOWERing hospitalized older adults to deprescribe sedative hypnotics: a pilot study. J Am Geriatr Soc. 2018;66(6):1186-9.

6. McKean M, Pillans P, Scott IA. A medication review and deprescribing method for hospitalised older patients receiving multiple medications. Intern Med J. 2016;46(1):35-42.

7. Scott IA, Hilmer SN, Reeve E, Potter K, Le Couteur D, Rigby D, et al. Reducing inappropriate polypharmacy: the process of deprescribing. JAMA Intern Med. 2015;175(5):827-34.

8. Potter EL, Lew TE, Sooriyakumaran M, Edwards AM, Tong E, Aung AK. Evaluation of pharmacist-led physician-supported inpatient deprescribing model in older patients admitted to an acute general medical unit. Australas J Ageing. 2019;38(3):206-10.

9. Kouladjian O'Donnell L, Sawan MJ. Deprescribing needs to be considered in the pharmacists' prescribing role. Med J Aust. 2020;212(3):141-e1.

10. Edey R, Edwards N, Von Sychowski J, Bains A, Spence J, Martinusen D. Impact of deprescribing rounds on discharge prescriptions: an interventional trial. Int J Clin Pharm. 2019;41(1):159-66.

11. Scott S, Clark A, Farrow C, May H, Patel M, Twigg MJ, et al. Deprescribing admission medication at a UK teaching hospital; a report on quantity and nature of activity. Int J Clin Pharm. 2018;40(5):991-6.

12. MacMillan TE, Kamali R, Cavalcanti RB. Missed opportunity to deprescribe: docusate for constipation in medical inpatients. Am J Med. 2016;129(9):1001.e1-7.

13. Reeve E. Deprescribing tools: a review of the types of tools available to aid deprescribing in clinical practice. J Pharm Pract Res. 2020; 50(1):98-107.
Lisa Kouladjian O'Donnell, BMedSc(Hons), MPharm, PhD Departments of Clinical Pharmacology and of Aged Care Kolling Institute of Medical Research, Royal North Shore Hospital St Leonards, New South Wales, Australia

Northern Clinical School, Faculty of Medicine and Health The University of Sydney

Sydney, New South Wales, Australia

Competing interests: Lisa Kouladjian O'Donnell is a member of the Australian Deprescribing Network Executive Committee (volunteer role). No other competing interests were declared.

\section{THE "CON" SIDE}

Two of every 3 Canadians over the age of 65 years take 5 or more prescription medications, often referred to as polypharmacy. ${ }^{1}$ Polypharmacy can be appropriate and necessary, occurring as a result of multimorbidity combined with an extended lifespan. However, more than $30 \%$ of older Canadian adults are taking 1 or more potentially inappropriate medications (PIMs; defined as medications for which the risk outweighs the benefit). ${ }^{1}$ Deprescribing is the process of reducing or discontinuing inappropriate medications, with the goal of reducing risk and negative outcomes in older adults. ${ }^{2}$ Research focused on deprescribing is growing; however, the current literature is heterogeneous, involving different types of interventions, providers, and contexts, with variable efficacy., ${ }^{3,4}$

Several points of opportunity for deprescribing have been described in the literature. ${ }^{5-7}$ Hospital admission, in particular, is thought to provide an ideal opportunity for deprescribing, because the patient's medication history is reviewed, clinicians are working in a collaborative environment, and patients and families are engaged in the process during the hospital stay. ${ }^{3,4,8}$ However, there are significant challenges to deprescribing in the hospital setting that limit this opportunity. ${ }^{5-7}$ First, hospital admission is often the result of an acute issue, making deprescribing of long-term medications less of a priority. Indeed, changes to regular medications during hospitalization could cause new symptoms or a change in condition, which could influence monitoring of recovery from the acute issue. A 2018 narrative review determined that more research was required regarding clinicians' safety concerns related to deprescribing, such as withdrawal events and re-emergence of a condition. ${ }^{9}$

The time needed for deprescribing of certain medications (e.g., need for tapering) and continuity of care in the context of short admissions are also of concern. Given the economic costs, as well as the known risks during a hospital stay (e.g., errors, infection, deconditioning), lengthening a hospital stay to allow for deprescribing is unlikely to be appropriate. One in-hospital study, published in 2018, showed that hospital admission is itself a risk factor for prescribing of a PIM. ${ }^{10}$

In addition, issues related to fragmentation of care are exacerbated in hospital. These issues include difficulties 
accessing medical and/or medication history and the various specialties and clinicians involved in past and present care. Inability to access a patient's complete history limits the possibility of identifying whether a medication is inappropriate and therefore suitable for deprescribing. As well, reluctance to question what a colleague has prescribed may lead to hesitancy among clinicians to initiate the deprescribing process or review the need for a medication.,

Guidelines, along with diagnoses, test results, and symptoms, are used by clinicians to guide decision-making; they also promote a prescribing culture and subsequent polypharmacy. Typically, guidelines provide information about when to initiate a medication, but often neglect to provide information about discontinuation. ${ }^{12}$ Although deprescribing tools and guidelines are emerging, there is as yet no consistent process for or guide to this process. Lack of confidence among prescribers, combined with an absence of reliable decision support, may lead to continuation of medications, as this may be perceived as safer than discontinuation. ${ }^{11,13}$ As well, the effect of decision support tools requires more research. An in-hospital study of a decision support tool, published in 2019, showed a statistically significant decrease in PIMs with use of the tool; however, the effect on clinically significant outcomes was unclear. ${ }^{4}$ Overall, the impact of in-hospital deprescribing on clinically important outcomes is unknown, because studies have not been powered to evaluate outcomes such as readmission and mortality. ${ }^{3}$ In an already strained health care system, in-hospital activities that improve clinical outcomes should be prioritized.

Patients' preferences and goals of care also play a role in deprescribing. In a survey of older inpatients, $89 \%$ were hypothetically willing to stop 1 or more of their regular medications. ${ }^{8}$ However, attempts to deprescribe in clinical trials have not shown the same rate of success. ${ }^{14,15}$ In a cross-sectional study published in $2018,39.7 \%$ of patients refused deprescribing in hospital, and none of the variables measured, including number of PIMs, predicted refusal. ${ }^{15}$ Overall, patient characteristics and factors leading to patients' refusal of deprescribing constitute an area for further exploration. Another in-hospital study, published in 2019, highlighted the importance of patient education and engagement in the deprescribing process. ${ }^{7}$ The ability to discuss and ascertain patient preferences to drive appropriate deprescribing may be diminished in hospital, because there is no previously established relationship between patients and their care providers. The patient's level of trust and the physician-patient relationship are likely to be hugely influential in the success of deprescribing. ${ }^{8,16}$ Additionally, for patients experiencing an acute event or an otherwise significant point in their health care journey, it is not clear whether preferences expressed while in hospital will fluctuate or match preferences after discharge.

Transitions of care are particularly concerning and can affect the success of deprescribing. ${ }^{17,18}$ Lack of follow-up and absence of assumption of responsibility for patients in whom deprescribing has been initiated, especially those without a family doctor, are issues for prescribers. ${ }^{3,7}$ The literature shows that deprescribing efforts are often not sustained after hospital discharge, as approximately $25 \%$ of ceased medications are restarted within the following year. ${ }^{19}$ This often occurs even if the medication was discontinued because of an adverse effect or as part of a comprehensive assessment. Problems with transfer of information and the involvement of multiple health care providers are cited as possible causes for resumption of medications; however, the reasons for re-prescription of ceased medications require further research. ${ }^{19,20}$ Therefore, without appropriate communication channels following discharge, deprescribing during hospitalization may be futile and not a valuable use of time and resources.

Evidence exists of the barriers to deprescribing in hospital; for example, an evaluation of deprescribing in the hospital setting, published in 2018 , showed that only $4 \%$ of patients had a medication deprescribed..$^{21}$ It has also been shown that deprescribing in hospital tends to be reactive, not proactive. ${ }^{21}$ Because of these barriers, further research is required before hospitalization can be considered an "ideal" setting for deprescribing. Nonetheless, clinicians should make the most of every opportunity to increase patients' and clinicians' awareness of deprescribing. Education of patients and clinicians and initiation of discussions about deprescribing could certainly start in the hospital, as could identification of PIMs that should be reviewed by the primary care team. Communication and continuity during transitions of care are key, and discussions should be continued after discharge, with patients, families, and health care providers becoming informed and engaged in shared decision-making.

\section{References}

1. Drug use among seniors in Canada, 2016. Canadian Institute for Health Information; 2018 [cited 2020 Apr 14]. Available from: https:// www.cihi.ca/sites/default/files/document/drug-use-among-seniors2016-en-web.pdf

2. Reeve E, Gnjidic D, Long J, Hilmer S. A systematic review of the emerging definition of 'deprescribing' with network analysis: implications for future research and clinical practice. Br J Clin Pharmacol. 2015;80(6):1254-68.

3. Thillainadesan J, Gnjidic D, Green S, Hilmer S. Impact of deprescribing interventions in older hospitalised patients on prescribing and clinical outcomes: a systematic review of randomised trials. Drugs Aging. 2018;35(4):303-19.

4. McDonald E, Wu P, Rashidi B, Forster A, Huang A, Pilote L, et al. The MedSafer study: a controlled trial of an electronic decision support tool for deprescribing in acute care. J Am Geriatr Soc. 2019;67(9):1843-50.

5. Scott IA, Hilmer SN, Reeve E, Potter K, Le Couteur D, Rigby D, et al Reducing inappropriate polypharmacy: the process of deprescribing. JAMA Intern Med. 2015;175(5):827-34.

6. Ailabouni N, Nishtala P, Mangin D, Tordoff J. Challenges and enablers of deprescribing: a general practitioner perspective. PLoS One. 2016;11(4):e0151066.

7. Baysari M, Duong M, Zheng W, Nguyen A, Lo S, Ng B, et al. Delivering the right information to the right person at the right time to facilitate deprescribing in hospital: a mixed methods multisite study to inform decision support design in Australia. BMJ Open. 2019;9(9):e030950. 
8. Reeve E, Wiese M, Hendrix I, Roberts M, Shakib S. People's attitudes, beliefs, and experiences regarding polypharmacy and willingness to deprescribe. J Am Geriatr Soc. 2013;61(9):1508-14.

9. Reeve E, Moriarty F, Nahas R, Turner J, Kouladjian O’Donnell L, Hilmer S. A narrative review of the safety concerns of deprescribing in older adults and strategies to mitigate potential harms. Expert Opin Drug Saf. 2018;17(1):39-49.

10. Pérez T, Moriarty F, Wallace E, McDowell R, Redmond P, Fahey T. Prevalence of potentially inappropriate prescribing in older people in primary care and its association with hospital admission: longitudinal study. BMJ. 2018;363:k4524.

11. Scott S, Twigg M, Clark A, Farrow C, May H, Patel M, et al. Development of a hospital deprescribing implementation framework: a focus group study with geriatricians and pharmacists. Age Ageing. 2019;49(1):102-10.

12. Okeowo D, Patterson A, Boyd C, Reeve E, Gnjidic D, Todd A. Clinical practice guidelines for older people with multimorbidity and lifelimiting illness: what are the implications for deprescribing? Ther $A d v$ Drug Saf. 2018;9(11):619-30.

13. Potter K, Flicker L, Page A, Etherton Beer C. Deprescribing in frail older people: a randomised controlled trial. PLoS One. 2016;11(3):e0149984.

14. Scott S, Clark A, Farrow C, May H, Patel M, Twigg M, et al. Attitudinal predictors of older peoples' and caregivers' desire to deprescribe in hospital. BMC Geriatr. 2019;19:Article 108.

15. Komagamine J, Sugawara K, Hagane K. Characteristics of elderly patients with polypharmacy who refuse to participate in an in-hospital deprescribing intervention: a retrospective cross-sectional study. BMC Geriatr. 2018;18:Article 96.

16. Reeve E, Low L, Hilmer S. Beliefs and attitudes of older adults and carers about deprescribing of medications: a qualitative focus group study. Br J Gen Pract. 2016;66(649):e552-60.

17. Viktil K, Blix H, Eek A, Davies M, Moger T, Reikvam A. How are drug regimen changes during hospitalisation handled after discharge: a cohort study. BMJ Open. 2012;2(6):e001461.

18. Daliri S, Bekker CL, Buurman BM, Scholte op Reimer WJM, van den Bemt BJF, Karapinar-Çarkit F. Barriers and facilitators with medication use during the transition from hospital to home: a qualitative study among patients. BMC Health Serv Res. 2019;19:Article 204.

19. Lampela P, Hartikainen S, Lavikainen P, Sulkava R, Huupponen R. Effects of medication assessment as part of a comprehensive geriatric assessment on drug use over a 1-year period: a population-based intervention study. Drugs Aging. 2010;27(6):507-21.

20. van der Linden CMJ, Kerskes MCH, Bijl AMH, Maas HAAM, Egberts ACG, Jansen PAF. Represcription after adverse drug reaction in the elderly: a descriptive study. Arch Intern Med. 2006;166(15):1666-7.

21. Scott S, Clark A, Farrow C, May H, Patel M, Twigg M, et al. Deprescribing admission medication at a UK teaching hospital; a report on quantity and nature of activity. Int J Clin Pharm. 2018;40(5):991-6.

Marci E Dearing, BSc(Pharm), PharmD, ACPR

QEII Health Sciences Centre, Nova Scotia Health Authority

Halifax, Nova Scotia

Competing interests: For research that involved deprescribing but was not related to the current article, Marci Dearing participated in a project that received a grant from the Canadian Frailty Network. No other competing interests were declared. 